\title{
JETDE Preface
}

\author{
Marcus D. Childress
}

I am proud to introduce this issue of the Journal of Educational Technology and Development Exchange. This compendium of scholarly writing illustrates the breadth, depth, and diversity of work in the area of teaching and learning. The articles in this issue provide a glimpse into the ongoing research and scholarly dialogue in educational technology.

Ying Wang Shen presents a case study examining the application of blended learning in accelerated post-baccalaureate teacher education at the program level. Using mixed methods data collection analysis, Shen's study highlights the benefits and challenges of applying blended learning in teacher education. Shen ends by making recommendations for applying blended learning in teacher education at the program level.

Weiyuan Zhang shareshis view of a new, 3rd generation of e-learning by first reviewing the developmental stages of e-learning: a) one-way transmission, b) interactive, c) comprehensive mode. Proposed strategies in establishing this new generation of elearning were shared, including platform specifications, course development strategies, and staff development approaches. The article illustrates how e-learning has matured to a level where we can now concentrate on developing more efficient and rich learning environments.

Guoqiang Cui provides a comprehensive review of social presence assessment. The article provides insight into the historical development of social presence assessment. Cui introduces subjective and objective measures of social presence, criticisms towards current social presence measures, and recommendations for future development of social presence measurement tools. The article serves as a good starting point for those wishing to further investigate the construct of social presence.

Liang Yu and Rong-Huai Huang offer a a quasi-experiment study that examines the effects of individual characteristics (gender and learning style), group characteristics (task type and group production), and their interaction effect on learners' knowledge acquisition and acquirement of collaborative skills. The group with scripted task and the group with scripted task and sequence demonstrated higher levels of knowledge acquisition and collaborative skills than the group with unscripted task. Significant differences in the effect of scripted task on knowledge acquisition and collaborative skills were found among students with surface approach, strategic approach and deep approach.Although scripted task group performance was not significantly different from the group with scripted task and sequence on knowledge acquisition and collaborative skills, the results suggest that collaborative learning researchers should pay closer attention to facilitating the enhancement of the collaborative skills.

Using a case-study approach, TaralynnHartselldescribes the process of creating digital videos as part of a graduate student reflection assignment in an instructional design course. The article begins by highlighting the research and benefits of using digital video and integrating a digital storytelling framework. Hartsell then outlines the use of digital 
video reflections for authentic assessment.Benefits, limitations, recommendations, and toolsfor creating digital video reflections are shared. This paper supports the value and importance of using authentic assessments such as digital videos in the classroom and will be of special interest to those wishing to integrate digital video reflections into their assessment routine.

Jingjing Zhang offers a qualitative research study that involved semi-structured interviews of experienced researchers from the disciplines of science, social sciences, and the humanities. Zhang presents a qualitative research study involving 24 senior researchers. The study found that a new type of trust (distributed trust) increasingly emerges in distributed research. As a prerequisite for generating mediated dialogue conducive to research exchange, distributed trust is likely to be developed in mediated dialogue at a distance. Zhang's study supports the idea that largely interdisciplinary and interconnected networks of distributed research are conducive to academic discourse.

SICET's Volume 6, Number 1, issue of JETDE continues a tradition of interesting and well-edited articles; scholarly writing that shares evidence-based research and practice in the field of educational technology. I am confident that you will find the reports both stimulating and beneficial. 